\title{
An Adaptive Level Damped Algorithm based on PINS for Ship
}

\author{
Pei Yu, GongLiu Yang \\ School of Instrument Science \& opto-electronics Engineering \\ Beijing University of Aeronautics and Astronautics \\ Beijing, China \\ e-mail: sally1130wallace@gmail.com \\ bhu17-yang@139.com
}

\begin{abstract}
The inertial navigation system error caused by sea current and log error greatly oscillates. In order to evaluate the effectiveness of damping attitude, the vehicle's movement should be detected in real-time. For this reason, a novel adaptive level damped algorithm was presented in this paper. According to the characteristics of the movement of the ship, this algorithm has determined when to turn into the damp loop according to the change of acceleration. Theoretical analysis and Simulations results show that the adaptive level damped algorithm could damp most of the Schuler and Foucault oscillations period, and efficiently improve the precision of the PINS on ships.
\end{abstract}

Keyword:adaptive control;level damp;Platform Inertial navigation system;schuler cycle oscillation

\section{INTRODUCTION}

Inertial navigation system is a completely self-contained system which is low cost and kind of simple structure type. It has no influence by a variety of climate conditions and external interference. Long-submerged nuclear-powered submarines needs INS to meet high requirements for hidden which particularly important. It mainly used for the carriage information which outputs speed and position information for submarine [1]. PINS system needs to provide accurate navigation information for several days for submarine $[2,3]$. Due to the presence of a shock error and the gyro random drift accumulation, the no-damped inertial navigation system which meet the need of Schuler period greatly reduced the resetting cycle and working hours in order to improve the precision of the PINS on ships.

However, considering the perspective of military applications, GPS signal is not quite credibility during wartime and cannot be used underwater [4,5]. When the system is in case of small acceleration, the traditional platform inertial navigation system joining damping network cannot effectively inhibited the Schuler oscillation period of the system.

In this paper, the adaptive level algorithm monitors the characteristics of the movement of the ship by switching the system between no-damped and damped mode to ensure the inertial navigation system work independently. Simulation results verified the effectiveness of the algorithm.

\section{THE DESIGN OF DAMPING LOOP OF LEVEL VELOCITY AND PARAMETER}

The no-damped inertial navigation system is a critical stability system. The error of the system is periodic oscillation and the amplitude which is not attenuated. Besides the longitude error increased over time in the role of the constant error source, the rest six error parameters(two speed error, three attitude error and latitude error) can be maintained on a limited amplitude periodic oscillations. The system meets the need of the Schuler period, but it will cause three kinds of error period under disturb of many factors. The three kinds of error period are called separately the 84.4 minutes Schuler period, the 24-hour earth period and the Foucault period varies with latitude which phase modulate with Schuler period.

The three kinds of surge errors are bad for the inertial navigation system fixed on the warship. Nowadays the precision of the fiber gyroscope is low-level which deduce the velocity error increase quickly and then influence the precision of the attitude and heading. So it is very necessary to add the damping network into the system.

\section{A. The Design of level damping network}

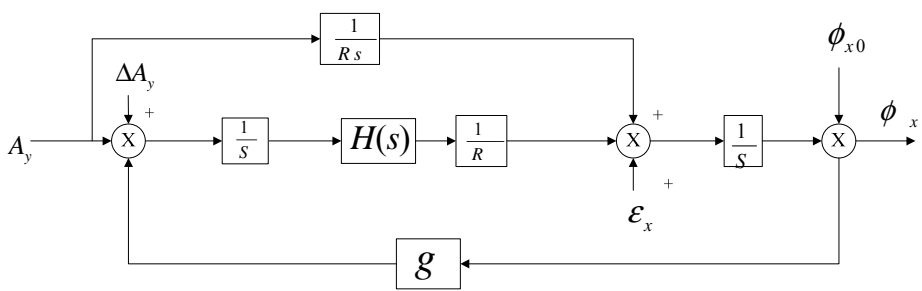

Figure 1. Structrure of System North Damp loop

As shown in Figure 1, Where $A_{y}$ is acceleration of north, $\Delta A_{y}$ is null position error of North accelerometer, $V_{y}$ is the North velocity, $\mathcal{E}_{x}$ is East gyro drift, $\phi_{x 0}$ is initial East misalignment angle of the platform, $R$ is earth radius, $g$ is acceleration of gravity, $\phi_{x}$ is east misalignment angle of the platform, $\frac{1}{S}$ is integral operator, $H(s)$ is the damping 
network in PINS circuit which are used to damp Schuler period and the Foucault period.

From the Figure 1 we can see, when $H(s)$ is not equal to 1 , i.e. the system select level damping network that the PINS start to damp. Due to $\Delta A_{y} \varepsilon$ and $\alpha_{0}$, level slope angle error which produced by system would be gradually damped. But acceleration and speed produce the error components of navigation parameters. Traditional damp algorithm was used in internal platform, which content is: when the system acceleration is small enough, system use velocity to damp gyroscope control loop for adjusting the system attitude.

The choosing principles of damping network are as follows:

a) It's capable of damp the Schuler oscillation period;

b) It has a smaller influence on system,i.e. the inertial navigation system has minimum sensitivity of ship motion;

c) From the view of the inertial navigation system error, when the system under steady state, $H(s)$ is the magnification factor. To make the sensitivity of the PINS the least,we have to set $H(s)$ close to 1 . Also $H(s)$ provide positive phase shift nearby the Schuler angular frequency $\omega_{x}$.

To guarantee the stability of the system, the form of damping network:

$$
H(s)=1+\frac{A}{s+W_{1}}+\frac{B}{s+W_{2}}
$$

The system secular equation is

$$
1+H(s) g / R s^{2}=0
$$

\section{B. The Design Of The Parameters In The Level Damping Network}

Using the north circuit as an example, in the Schuler first integration and the second integration, we add horizontal damping network $H(s)$. The Schuler circuit is a vibration system whose rank is 2 which resist Schuler period and Foucault period. In actual application, the system dynamics is an important technical indicator and the effect of errors in the form of the system are much more complex than the assumptions in the above theoretical analysis. Therefore, we select parameters through trial and error on the basis of analysis. We substitute formulation (1) into the system secular equation and then become:

$$
\begin{aligned}
& s^{4}+\left(W_{1}+W_{2}\right) s^{3}+\left(W_{1} W_{2}+\omega_{n}^{2}\right) s^{2}+ \\
& \omega_{n}^{2}\left(W_{1}+W_{2}+A+B\right) s \\
& +\omega_{n}^{2}\left(W_{1} W_{2}+A W_{2}+B W_{1}\right)=0
\end{aligned}
$$

We hypothesis two poles $S_{1,2}=-\sigma \pm j \omega_{d}$, extra poles are $s_{3}=-n_{1} \sigma ; s_{4}=-n_{2} \sigma \quad n_{1}, n_{1} \geq 5$

From the view of control theory

$$
\omega_{d}=\omega_{n} \sqrt{1-\mu^{2}}, \sigma=\omega_{n} \xi
$$

The system secular equation turns into

$$
\begin{aligned}
& s^{4}+\left(n_{1}+n_{2}+2\right) \xi \omega_{n} s^{3}+\left\{\left[n_{1} n_{2}+2\left(n_{1}+n_{2}\right)+1\right] \xi^{2} \omega_{n}^{2}+\right. \\
& \left.\omega_{n}^{2}\left(1-\xi^{2}\right)\right\} s^{2}+\left[\left(2 n_{1} n_{2}+n_{1}+n_{2}\right) \xi^{3} \omega_{n}^{3}+\right. \\
& \left.\left(n_{1}+n_{2}\right)\left(\xi-\xi^{3}\right) \omega_{n}^{3}\right] s+ \\
& {\left[n_{1} n_{2} \xi^{4} \omega_{n}^{4}+n_{1} n_{2} \omega_{n}^{4}\left(\xi^{2}-\xi^{4}\right)\right]=0}
\end{aligned}
$$

Based on equal to corresponding term:

$$
\left\{\begin{array}{l}
W_{1}+W_{2}=\left(n_{1}+n_{2}+2\right) \xi \omega_{n} \\
W_{1} \bullet W_{2}=-\omega_{n}^{2}+\left[n_{1} n_{2}+2\left(n_{1}+n_{2}\right)+1\right] \xi^{2} \omega_{n}^{2}+\omega_{n}^{2}\left(1-\xi^{2}\right) \\
W_{1}+W_{2}+A+B=\frac{2\left(n_{1}+n_{2}+2\right) \xi^{3} \omega_{n}^{3}+\left(n_{1}+n_{2}\right)\left(\xi-\xi^{3}\right) \omega_{n}^{3}}{\omega_{n}^{2}} \\
W_{1} \bullet W_{2}+A \bullet W_{2}+B \bullet W_{1}=\frac{2\left(n_{1}+n_{2}+2\right) \xi^{3} \omega_{n}^{3}+\left(n_{1}+n_{2}\right)\left(\xi-\xi^{3}\right) \omega_{n}^{3}}{\omega_{n}^{2}}
\end{array}\right.
$$

According to system pendulum stability

$$
\left|n_{1}-n_{2}\right| \geq 9
$$

Assuming a b c d as follows:

$$
\left\{\begin{array}{l}
a=-\left(n_{1}+n_{2}+2\right) \xi \omega_{n} \\
b=-\omega_{n}^{2}+\left[n_{1} n_{2}+2\left(n_{1}+n_{2}\right)+1\right] \xi^{2} \omega_{n}^{2}+\omega_{n}^{2}\left(1-\xi^{2}\right) \\
c=\frac{-\left(2 n_{1} n_{2}+n_{1}+n_{2}\right) \xi^{3} \omega_{n}^{3}+\left(n_{1}+n_{2}\right)\left(\xi-\xi^{3}\right) \omega_{n}^{3}}{\omega_{n}^{2}} \\
d=\frac{n_{1} n_{2} \xi^{4} \omega_{n}^{4}+n_{1} n_{2} \omega_{n}^{4}\left(\xi^{2}-\xi^{4}\right)}{\omega_{n}^{2}}
\end{array}\right.
$$

The solutions of equation are as follows:

$$
\begin{aligned}
& W_{1}=\frac{2 b}{a+\sqrt{a^{2}-4 b}} ; W_{2}=\frac{b}{W_{1}} ; \\
& A=\frac{(c-a) W_{1}-(d-b)}{W_{1}-W_{2}} ; B=\frac{-A W_{2}}{W_{1}}
\end{aligned}
$$

We finally set damping parameter are as follows $\xi=0.707$

$$
n_{1}=5, n_{2}=14, \omega_{n}=\sqrt{g / R}=1.24 \times 10^{-3}(\mathrm{rad} / \mathrm{s})
$$

\section{THE DESIGN OF ADAPTIVE LEVEL DAMPING LOOP}

Accelerometer measured linear accelerated velocity parameters (inertial force), $\Delta A_{0}$ is null position error of (3) accelerometer, $\omega_{a}$ is white-noise random drift of 
accelerometer, $\nabla_{r}$ is first order Markov process, and it is assumed the same as the three axis.

Accelerometer error model[6] is as follows:

$$
\nabla^{b}=\Delta A_{0}+\nabla_{r}+\omega_{a}
$$

Where $\nabla_{r}$ is first order Markov process which mathematical model is as follows:

$$
\dot{\nabla}_{r}=-\frac{1}{T_{a}} \nabla_{r}+\omega_{r}
$$

Where $T_{a}$ is correlation time, $\omega_{r}$ is the Markov processdriven white noise. As first order Markov process with zero errors by the side of white-noise random drift and null position error is small enough to ignore its impact. We add adaptive judgment process in the level damping network according to the real-time characteristics of the movement of the ship. When the accelerometer output exceeds the threshold value range, the level damping network failure, only operate in tradition resolution process; otherwise, use the level damping network.

\section{SIMULATION}

We supposed the simulation conditions are:

a) constant drift of gyro is $\varepsilon_{x 0}=0.003\left(^{\circ}\right) / h ; \varepsilon_{y 0}=0.005\left(^{\circ}\right) / h ; \varepsilon_{z 0}=0.01\left(^{\circ}\right) / h$;

b) null position error of accelerometer is $\Delta A_{x 0}=\Delta A_{y 0}=5.0 \times 10^{-6} \mathrm{~g}\left(\mathrm{~m} / \mathrm{s}^{2}\right)$;

c) random drift of accelerometer is Gaussian white noise with zero-mean and standard deviation of $10^{(-7)} \times g\left(\mathrm{~m} / \mathrm{s}^{2}\right)$;

d) misalignment angle to the platform is $\alpha_{0}=3^{\prime}, \beta_{0}=\gamma_{0}=5^{\prime}$;

e) other souces of error all set 0 ;

f) initial state all set 0 ;

g) $0 \sim 12 \mathrm{~h}:$ static status;

h) $12 \mathrm{~h} \sim 48 \mathrm{~h}$ : variable motion status;

i) 48h 2h: uniform motion status;
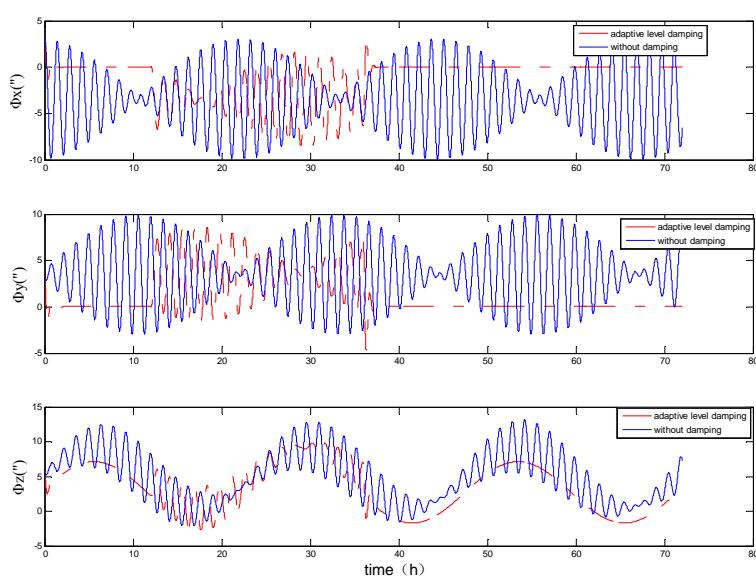

Figure 2. Attitude Error Without Damping and With Adaptive Damping
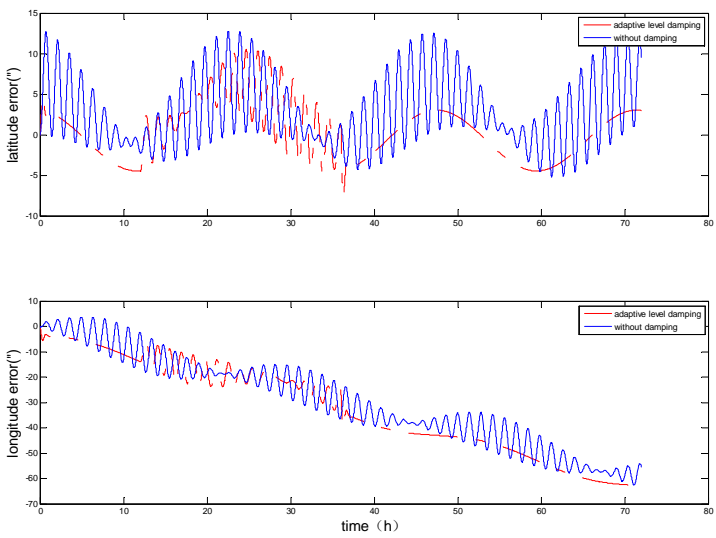

Figure 3. Position Error Without Damping and With Adaptive Damping
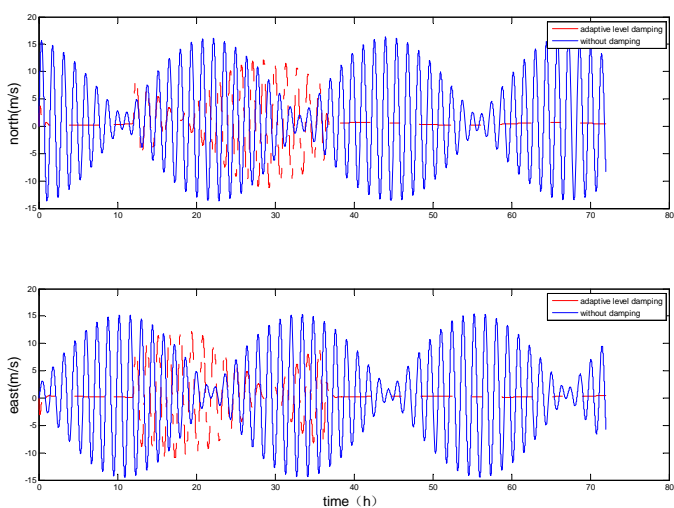

Figure 4. Velocity Error Without Damping and With Adaptive Damping

Fig 2, Fig 3, Fig 4 is attitude error, position error and velocity error without damping respectively and with adaptive level damping respectively. The algorithm which 
determined the performance of ship current moment based on platform inertial navigation system to switch between the no-damping mode and damping mode to ensure the accuracy and independent of the system.

As we can see from Fig 2, the system horizontal errors have been greatly improved, though no greater improvement in the azimuth error which only suppress the Schuler oscillation period. However, the overshoot errors produced by mode switch from non-damping to damping increased which remains to be further validation. From Fig 3, the accuracy of latitude error as well as heading error only suppress Schuler oscillation period. Fig 4 shows the velocity errors with adaptive level damp have been improved compared to the state before no-damped.

Known from simulation and sea test, when sea current and $\log$ error greatly oscillates, the systematic error is the minimum under no-damped state. Nevertheless the system work in critical mode, in case of interfere system error easily divergence. So working in long working hours submarine rarely use in this way. On the other hand, single level damped algorithm worked under sea current and log error greatly oscillates. The attitude error, velocity error and the position error have large fluctuations caused by the so-called "bulge" phenomenon. So the adaptive level damp is a good way to inhibit the Schuler error oscillation period and the Foucault error oscillation period.

\section{CONCLUSION}

In this paper, we have presented a novel design of adaptive level damping loop algorithm which determined the performance of ship current moment based on platform inertial navigation system to switch between the no-damping mode and damping mode to ensure the accuracy of the system and autonomy. However, when faced with the shock waves and other interference, the robustness of the program remains to be further validation. Simulation results show that the algorithm can significantly inhibit Schuler oscillation period of inertial navigation system, effectively improve the dynamic performance of the INS with a good prospect of application and promotional value in use.

\section{ACKNOWLEDGMENT}

This research was supported by Science and Technology on Inertial Laboratory and Fundamental Science on Novel Inertial Instrument \& Navigation System Technology Laboratory.

\section{REFERENCES}

[1] FJ.QIN, A.LI, XU, F.ZHANG,"Horizontal inner damping method with continuously adjustable parameter for inertial navigaiton system",Journal of Chinese Inertial Technology.Vol.19 No.3,Jun 2011

[2] Dorf R C,Bishop R H.Modern control systems[M].12 ${ }^{\text {th }}$ Edition .New Jersey:Prentice Hall Press,2010:98-108

[3] Weston J L,Titterbn D H.Modern inertial navigation technology and its application [J].Electronics and Communication Engineering Journal,2000(8):49-64

[4] Vanderwerf K,Schuler pumping of inertial velocity errors due to gravity anomalies along a popular North Pacific ariway[C]

[5] R.E. Mayagoitia, A.V. Nene, P.H. Veltink, Accelerometer and rate gyroscopes measurement of kinematics: an inexpensive alternative tooptical motion analysis systems, J. Biomech. 35 (2002) 537-542.

[6] C.V.C. Bouten, K.T.M. Koekkoek, M. Verduin, R. Kodde, J.D.Janssen, A triaxial accelerometer and portable data processing unitfor the assessment of daily physical activity, IEEE Trans. Biomed.Eng. 44 (3) (1997) 136-147. 